
of Engineers ${ }_{\circledast}$

Engineer Research and Development Center

Geospatial Technology Research and Development Program

Using Terminal Services to Serve Geospatial Software and Data Resources to Corps Project Offices

Mark R. Graves

August 2003

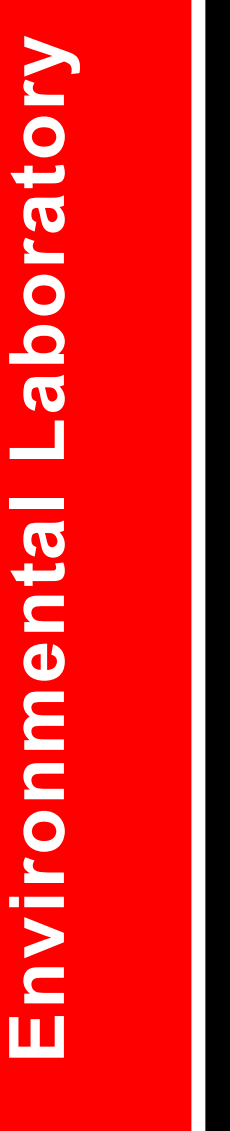

Approved for public release; distribution is unlimited. 


\section{Using Terminal Services to Serve Geospatial Software and Data Resources to Corps Project Offices}

Mark R. Graves

Environmental Laboratory

U.S. Army Engineer Research and Development Center

3909 Halls Ferry Road

Vicksburg, MS 39180-6199

Final report

Approved for public release; distribution is unlimited 


\begin{abstract}
Advances in geographic information systems (GIS) are causing the technology to no longer be considered a separate entity, but rather an integral component of the overall information technology infrastructure. Most GIS platforms are moving from simple file-based data structures to complex spatial geodatabases built within large-scale database platforms, such as Oracle. The move toward centralized databases has many ramifications for how geospatial tools and data are distributed throughout a complex organization such as the Corps of Engineers. Project offices, in particular, represent a special challenge due to the limitations of their network connections to the district offices. Windows Terminal Server technology represents one solution for serving these resources to project and field offices. This document addresses some of the technical issues related to the use of this technology and some of the advantages and limitations of such an approach.
\end{abstract}

DISCLAIMER: The contents of this report are not to be used for advertising, publication, or promotional purposes. Citation of trade names does not constitute an official endorsement or approval of the use of such commercial products. All product names and trademarks cited are the property of their respective owners. The findings of this report are not to be construed as an official Department of the Army position unless so designated by other authorized documents. 


\section{Contents}

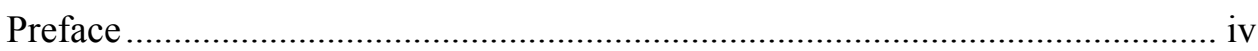

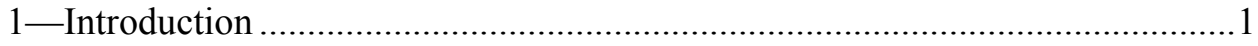

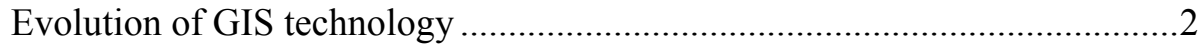

Disseminating Geospatial Data and Applications to Field Offices..................2

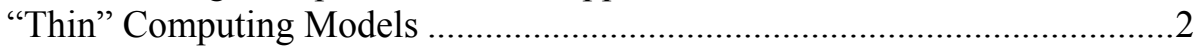

Service-Based Internet Mapping Applications......................................... 3

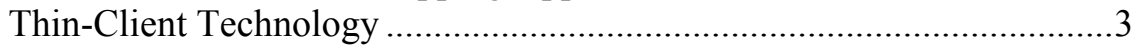

2 - Overview of Terminal Server Technology .................................................. 6

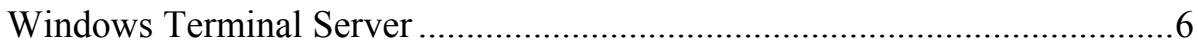

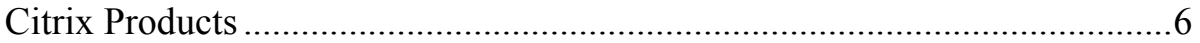

Advantages of a Windows Terminal Server Implementation .........................8

Disadvantages of a Windows Terminal Server Implementation......................9

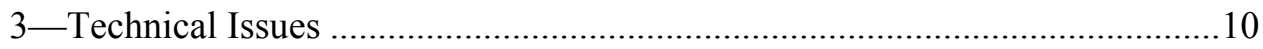

Server Sizing and Configuration.............................................................. 10

Windows and Virtual Memory Use....................................................10

Recommendations on Server Selection .................................................11

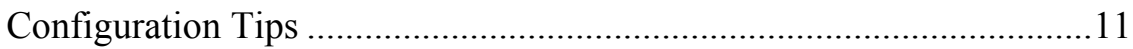

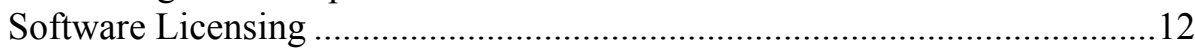

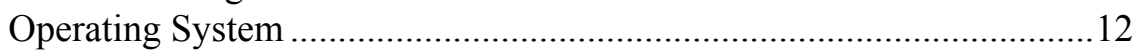

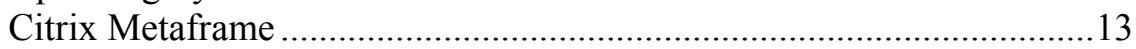

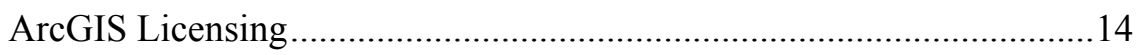

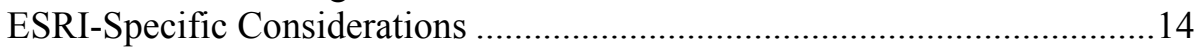

Terminal Server Support from ESRI ..................................................15

ArcGIS Installation on Terminal Servers .............................................15

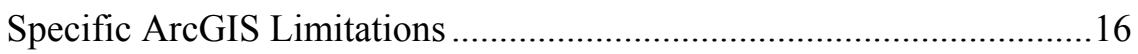

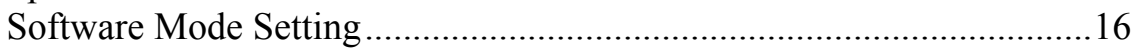

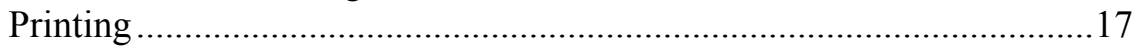

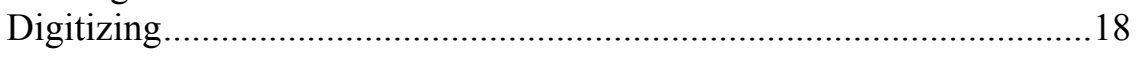

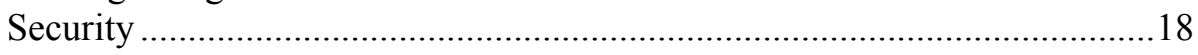

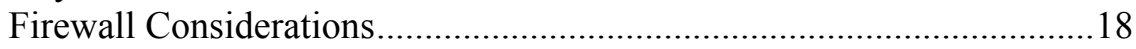

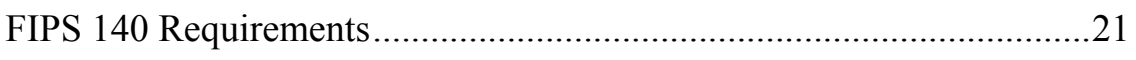

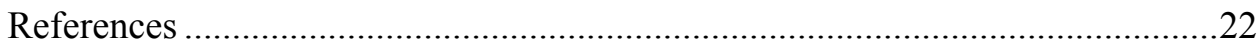

SF 298 


\section{Preface}

This report was prepared as part of the Geospatial Technology Research and Development Program Work Unit 36302, "Integrated Geospatial Technologies to Support Natural Resource Management." Research was conducted by the Environmental Laboratory (EL), U.S. Army Engineer Research and Development Center (ERDC), Vicksburg, MS. The research was sponsored by Headquarters, U.S. Army Corps of Engineers (HQUSACE), and was carried out during the period of January 2002 to December 2002.

This report was prepared by Mr. Mark R. Graves, Environmental Systems Branch (ESB), EL. The study was under the general supervision of Mr. Harold W. West, Chief, ESB; Dr. David J. Tazik, Chief, Ecosystem Evaluation and Engineering Division, EL; and Dr. Edwin A. Theriot, Director, EL. The HQUSACE Technical Monitors were Mr. M. K. Miles and Ms. Nancy J. Blyler. Reviews were provided by Dr. Linda P. Peyman-Dove and Mr. Scott G. Bourne, ESB.

Commander and Executive Director of ERDC was COL James R. Rowan, EN. Director was Dr. James R. Houston. 


\section{Introduction}

Fielding geospatial software tools, applications, and data throughout a district can be a costly and complex task. The goal is to provide the proper tools to decision makers while, at the same time, minimizing costs - including administration, maintenance, training, support, and upgrade expenses. These challenges are made more difficult by the fact that many Corps districts include field offices, which are often located in rural areas and connected to the main office by limited network connections.

Many field offices are now using geographic information system (GIS) software for a growing range of applications. While the use of this software is yielding increased efficiency and better management of Corps resources, it also comes at a cost. Many districts are now purchasing and maintaining more copies of software than are required. Field offices using these packages often require more expensive personal computers than they would otherwise need. Finally, advances in geospatial technology have resulted in a shift from file-based solutions to a database-oriented approach, which requires connection to a centralized relational database management system (RDBMS), such as Oracle. Field offices, with their limited bandwidth connection to the main district office, are prohibited from fully realizing the benefits from such a corporate approach to geospatial technology.

The use of terminal server technology provides one avenue to address some of these concerns. This report deals with specific issues that must be addressed with the use of Windows terminal services to support serving geospatial software and applications in a manner which would allow field offices to have access to the full range of geospatial tools. As many Corps offices are using the Environmental Systems Research Institute (ESRI) suite of geospatial applications, specific considerations for serving these applications in a terminal server environment are addressed.

While this document focuses mainly on geospatial resources and software issues, it should be understood that Corps districts can realize many additional benefits in deploying many other types of applications through a terminal server. Indeed, a terminal server can be used to serve almost any application that is now installed on individual PC's. For example, Microsoft Office applications, such as Word and Outlook, can be provided to end users through terminal server sessions, and users at remote sites, such as field offices, can recognize significant benefits in terms of stability and performance. 


\section{Evolution of GIS Technology}

When GIS technology was in its infancy, the software and spatial data required for geospatial applications usually resided on individual computers, manned by GIS technicians or specialists.

As the technology has evolved, geospatial applications have begun to permeate more and more of the business practices of the U.S. Army Corps of Engineers (USACE). In addition, client software has been developed that allows users with little GIS training to be able to use GIS data in their applications, especially for simple tasks such as displaying layers, overlaying various data types, or printing simple maps.

Most importantly, geospatial data itself has evolved from residing in proprietary file formats to being fully integrated into corporate RDBMS's such as Oracle. The Corps is now beginning to view geospatial data as a natural part of the organization's data assets and an integral component of the USACE corporate information infrastructure.

Because of these many changes, there has been the development of a move toward "enterprise GIS" solutions, with the goal of wisely managing and disseminating geospatial data and applications throughout the entire organization. The goal of these efforts is to appropriately protect the Corps' investment in geospatial data while putting the appropriate data and tools into the hands of decision makers.

\section{Disseminating Geospatial Data and Applications to Field Offices}

Field offices represent a unique challenge for enterprise GIS implementations. They are often connected to the district headquarters by very limited network lines, sometimes using $128 \mathrm{k}$ ISDN lines.

These connection limitations have required project or field offices to maintain copies of GIS databases. Updates to those databases by field personnel must be periodically reconciled with the "main" copy at the district office. In addition, the field offices must each maintain their own GIS software (including patches, extensions, etc.) and must possess computer equipment with sufficient power to run the software.

\section{“Thin” Computing Models}

To answer the problem of distributing geospatial information across limitedbandwidth networks, thin-client computing models and technologies have been developed. There are two basic types of "thin" approaches to computing. Both attempt to limit the amount of information that must cross the network, but they accomplish this in different ways and serve different purposes. These are described below. 


\section{Service-based Internet mapping applications}

The development of applications such as ArcIMS, GeoMedia Webmap, and the Minnesota Web Mapper represents approaches to disseminate geospatial information over the Web to client computers. Very small client applications, based on JAVA applets or ActiveX, are delivered to the client's Web browser. Figure 1 illustrates a service-based approach to the distribution of geospatial data using ESRI's Internet Mapping Software (IMS).

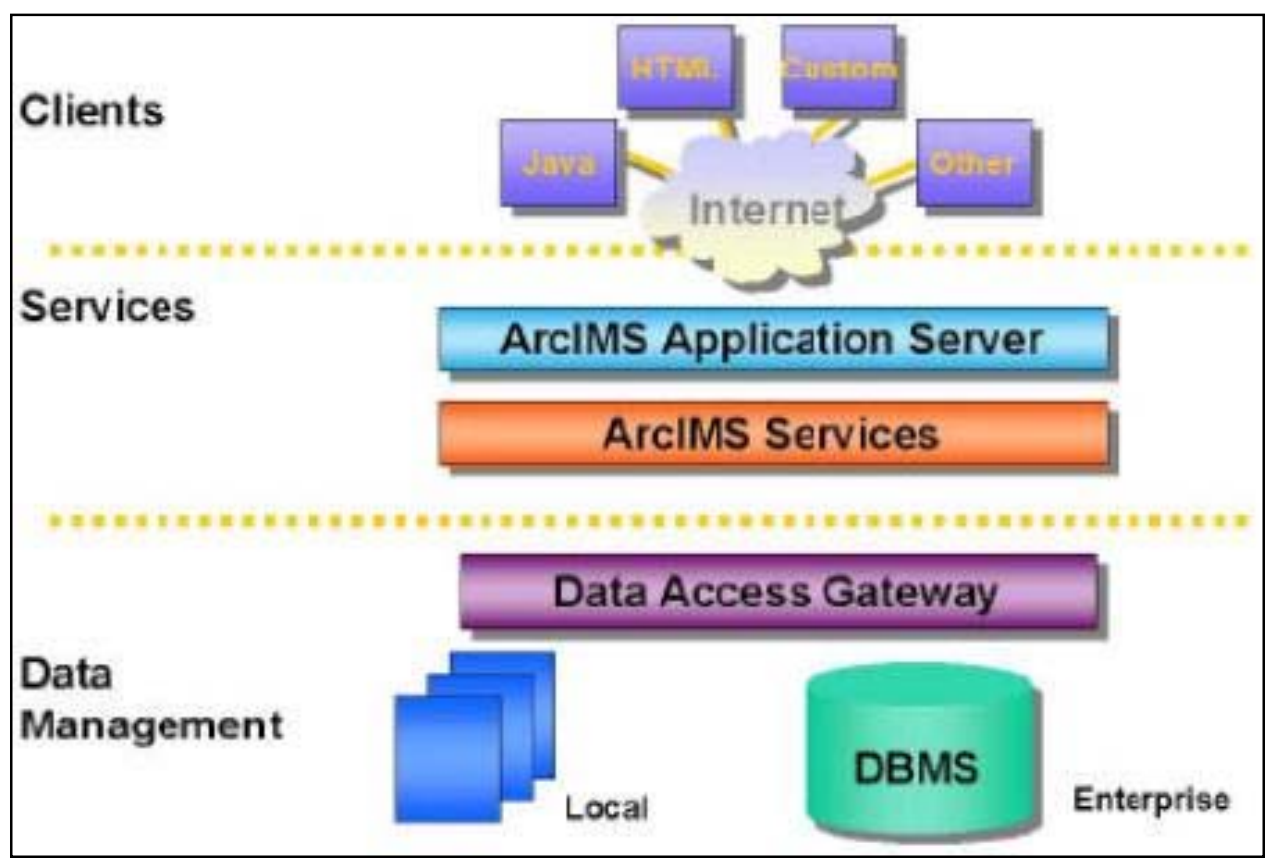

Figure 1. ArclMS software configuration (Peters 2003)

The information delivered to the client is restricted to what is required for display on the screen. Therefore, network bandwidth use is limited. However, the tiny client applications are usually rather limited. While these applications are good for providing access to end users with little or no GIS training, they cannot replace full-featured GIS applications such as ArcGIS.

\section{Thin-client technology}

Thin-client computing represents somewhat of a return to an old client/server computing paradigm. About a decade ago, most major computational and i/ointensive computer applications ran on large servers. Users connected to these applications through dumb ASCII terminals or through terminals running XWindows. With the development of more and more advanced PC's, these applications started to move to the desktop. GIS software followed this distributed-computing trend.

Unfortunately, this distributed-computing trend carries with it many negative aspects. The cost of maintaining and administering a large number of PC's throughout an organization is one of these. However, for implementing an 
enterprise GIS, it is not the main problem. The main problem is distributing GIS information throughout the organization to end users.

To implement an RDBMS-based GIS database and to manage that information in a manner similar to other enterprise databases, then the information must be centrally maintained and accessed directly by end users. However, geospatial databases are quite large, containing great amounts of graphical information. To support end users running GIS software in a distributed manner, all the graphical information must flow over the network to the client application. Users on a LAN can operate in this environment; however, for users at a field office, the network bandwidth is just not sufficient to support this model. To date, this has required the district office to distribute copies of GIS databases to field offices. This violates some of the basic rules of database management and will prohibit field offices from taking advantage of more advanced GIS data structures in the future.

The goal of the thin-client model is to centralize computing resources and recognize associated benefits of easier maintenance and less expensive upgrades, while maintaining the same quality of service for the end user that could be provided by a dedicated workstation. In a thin-client computing environment, although users can use PC's as terminals, users can move from full-featured computers to thin-client devices, lightweight machines primarily used for display and input and which require less maintenance and fewer upgrades. Organizations then provide computing services to their end users' thin clients from highpowered servers over a network connection. Server resources can be shared across many users, resulting in more effective utilization of computing hardware (Nieh et al. 2000).

The network requirements are limited due to the fact that only keystrokes, mouse clicks, and screen refreshes are transmitted between the server and the client (Figure 2).

This distributive system of information, compared with tools that are "standalone" or installed in a personal computer, offers the following advantages, among others:

- Sharing and exchange of data.

- Access to applications and tools for analysis and decision making for a more extensive public.

- Facilitates continued updating of information, helping to reduce redundancies (duplications) and improving access to databases.

- Facilitates the updating of applications and disclosed information.

The advantages (and limitations) of this approach are presented much more fully in the next section of this document. 


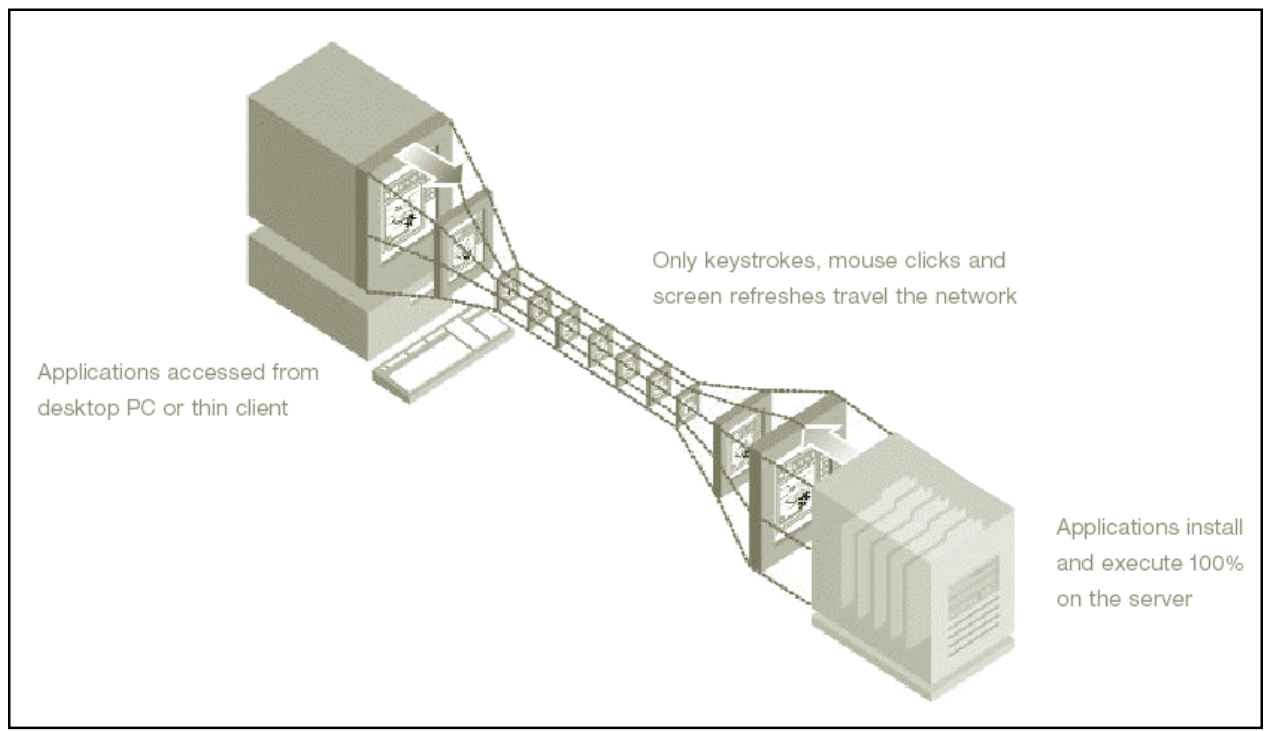

Figure 2. Overview of thin-client approach (Citrix Systems, Inc. 2002) 


\section{Overview of Terminal Server Technology}

Although Microsoft was a key player in the development of the distributedcomputing model, with the release of versions of their operating systems for larger, more powerful servers such as Windows NT 4.0, a need was recognized for a product that would serve a centralized computing model. Citrix Systems, Inc., had already produced a product (WinFrame) for NT 3.0 that would allow the operating system (OS) to support centralized computing. With NT Server 4.0, Microsoft purchased portions of the technology from Citrix, integrated the terminal server technology into the OS, and distributed a special version of NT called "NT 4.0 Server, Terminal Server Edition." Citrix continued to make products, such as MetaFrame, which enhances the core features provided by Microsoft.

\section{Windows Terminal Server}

With the release of Windows 2000, terminal services capabilities were included as an integral component of the OS with the Server, Advanced Server, and Datacenter Server versions. The fact that a special version of the OS was no longer required has hastened the acceptance of centralized computing models by many organizations.

Even though the terminal services included with Windows 2000 Server have greatly increased in features compared to earlier versions, most implementations of terminal server technology still benefit from the added features provided by Citrix MetaFrame XP.

\section{Citrix Products}

Citrix provides features that Microsoft has not added to the core terminal services. An example is the ability to do automatic drive remapping, which allows users to have access to the server drives as well as their local drives. Other Citrix features, such as Speedscreen 2, further compress the data stream traveling between the server and the client computer, making sessions even "thinner" and freeing up more network bandwidth. Other features, such as one-to-many and 
many-to-one shadowing, are very helpful when conducting training or instruction.

Load balancing is one of the most important features added by Citrix. As a terminal server implementation grows, additional servers can be added to the server "farm" to allow for supporting more users. The load-balancing support provided by Citrix is much more advanced than that provided in the base terminal services product provided by Microsoft.

Citrix also provides support, through their NFuse product (which is included with MetaFrame), for publishing applications via the Web. This can be a very useful tool for distributing applications throughout an organization.

Figure 3 illustrates the difference in features provided by Microsoft Terminal Services and the added features provided by Citrix MetaFrame.

\begin{tabular}{|c|c|c|}
\hline \multicolumn{3}{|c|}{ Feature Comparison } \\
\hline Client Features & MF & Win2K \\
\hline Manual Drive Redirection & $\mathrm{X}$ & $\mathrm{X}$ \\
\hline Persistent Bitmap Caching & $\mathrm{X}$ & $\mathrm{X}$ \\
\hline RSA Encryption & $\mathrm{X}$ & $\mathrm{X}$ \\
\hline Auto Printer Creation & $\mathrm{X}$ & $\mathrm{X}$ \\
\hline Clipboard Redirection & $\mathrm{X}$ & $\mathrm{X}$ \\
\hline System Sounds (beeps) & $\mathrm{X}$ & $\mathrm{X}$ \\
\hline Automatic Drive Redirection & $\mathrm{X}$ & \\
\hline COM Port Redirection & $\mathrm{X}$ & \\
\hline SpeedScreen 2 & $\mathrm{X}$ & \\
\hline Seamless Windows & $\mathrm{X}$ & \\
\hline 16-bit stereo (WAV, MIDI) & $\mathrm{X}$ & \\
\hline Video Support & $\mathrm{X}$ & \\
\hline Multimedia Bandwidth Control & $\mathrm{X}$ & \\
\hline Client Printer Management Utility & $\mathrm{X}$ & \\
\hline ReadyConnect & $\mathrm{X}$ & \\
\hline Business Recovery & $\mathrm{X}$ & \\
\hline Smart Card Support & $\mathrm{X}$ & \\
\hline Socks 4 \& 5 Support & $\mathrm{X}$ & \\
\hline Server Features & MF & Win2K \\
\hline TCP/IP Support & $\mathrm{X}$ & $\mathrm{X}$ \\
\hline Single Server Desktop Shadowing & $\mathrm{X}$ & $\mathrm{X}$ \\
\hline Randomized Load Balancing & & $\mathrm{X}$ \\
\hline Resource-based Load Balancing & $\mathrm{X}$ & \\
\hline Reconnect via Load Balancing & $\mathrm{X}$ & \\
\hline Cross Server Shadowing & $\mathrm{X}$ & \\
\hline Shadow Task Bar & $\mathrm{X}$ & \\
\hline One-to-Many Shadowing & $\mathrm{X}$ & \\
\hline Many-to-One Shadowing & $\mathrm{X}$ & \\
\hline Application Publishing & $\mathrm{X}$ & \\
\hline Program Neighborhood & $\mathrm{X}$ & \\
\hline Nfuse Web Portal Technology & $\mathrm{X}$ & \\
\hline Application Launch. \& Emb. (ALE) & $\mathrm{X}$ & \\
\hline Administrator Tool Bar & $\mathrm{X}$ & \\
\hline Anonymous User Support & $\mathrm{X}$ & \\
\hline Auto Client Update & $\mathrm{X}$ & \\
\hline Cross Domain / Subnet Mgmt. & $\mathrm{X}$ & \\
\hline IPX/SPX \& NetBios Support & $\mathrm{X}$ & \\
\hline Direct Asynch Dial-Up & $\mathrm{X}$ & \\
\hline
\end{tabular}

Figure 3. Comparison of Windows 2000 Server Terminal Services and Metaframe features (Citrix Systems, Inc. 2002) 


\section{Advantages of a Windows Terminal Server Implementation}

There are a number of significant advantages to the implementation of a terminal server approach to distributing applications throughout an organization. Some of the anticipated benefits are:

- Administration.

- Applications can be distributed quickly throughout the organization. Complex applications, such as ArcGIS, have a number of extensions and patches that must be periodically applied to make sure users have access to the latest versions. With a terminal server, all software, extensions, and patches will only have to be applied once - and not to multiple computers at both local and remote sites.

- Increased manageability and security of applications. The terminal server will make use of RAID storage and will be carefully maintained and backed up by an administrator. This will help protect the data and project files of users from catastrophic loss.

- Decreased need to upgrade client desktops computers as frequently. Since the speed of running applications will be as fast to a user running on an old computer as to one using the fastest available PC, the life cycle of existing computing resources will be essentially lengthened. This can result in significant savings to the district.

- Users provided with access to latest software features. ArcGIS users will be able to make use of multiuser geodatabases technology when the district decides to implement this method of storing GIS data. Implementation of multiuser geodatabases would not be possible to field offices without a terminal server implementation.

- Eliminate need to distribute data CD's to field users. Since users will be accessing data located on the district servers, there will be no need to create and distribute data CD's to field offices. The distribution and maintenance of multiple versions and copies of databases violates some basic principles of data management and can cause severe difficulties for any organization.

- Ease of providing technical support to end users. Through the use of session shadowing, GIS specialists at the district will be able to quickly answer questions of end users at the field offices or other users within the district building.

- Significant reduction in network bandwidth requirements. Since applications and data access are moved to a centralized computer room, network bandwidth usage is drastically reduced.

- User Environment.

- Fast access to district data resources. GIS users will be able to quickly access all data resources, including all raster imagery such as satellite images, digital ortho quads, etc. Anything available to GIS users at the district office will also be available - at the same access speed - to users at the field offices. 
- Improved application stability. Applications will be more stable because they will be kept up to date with the latest software patches. Also, a system administrator will be maintaining the terminal server.

- Technical support from district GIS specialists. Through the use of session shadowing, field users will be able to obtain quick technical support from GIS specialists located at the district office or from other GIS users at other field sites. Session shadowing allows both client sessions to see the same display and both to control the mouse and keyboard.

- Data protection. Data and important project files will be backed up on a frequent basis and will reside on RAID file systems - making it almost impossible to suffer a critical data loss.

- Matching operating environments. The application environment for any user using an application on the terminal server is identical. For example, the same ArcGIS extensions will be available to all users and, if a large data directory is maintained on the terminal server, all drive paths, environment variables, etc., will be identical. Therefore, any ArcMap documents saved on the machine could be opened by any other user (if sufficient permissions apply) without the need to edit the data connections or the use of any third-party software to handle such settings.

\section{Disadvantages of a Windows Terminal Server Implementation}

Although there are many advantages to a terminal server implementation, there are a few disadvantages. Among these are:

- Single point of failure. If a terminal server goes down - then there is no access to the GIS applications. A way around this is to adopt a server farm approach, in which the applications are mirrored on more than one server. This provides redundancy and allows for applications to remain available even when an individual server fails or is undergoing maintenance.

- System resources are shared. System resources will be shared by all users. Therefore, performance will be dictated by how powerful the server is and by how many simultaneous users are using the server. It is important in any terminal server implementation to carefully plan for anticipated loads.

- Feeling of loss of ownership. With the GIS applications moved to a central server, some users in the field may not be happy with the apparent loss of control of the applications. 


\section{Technical Issues}

\section{Server Sizing and Configuration}

To recognize acceptable performance, it is extremely important to purchase a server that possesses sufficient technical specifications to support the anticipated load. GIS software products, such as ArcGIS, use a great deal of memory. Each session of ArcMap or ArcCatalog can use as much as 60 to $70 \mathrm{MB}$ of memory. Therefore, it is important to purchase enough memory to limit paging. (Paging is what happens when a computer runs out of physical memory (or RAM).) When this happens, space on a hard disk, which has been set aside as "virtual memory," is used in place of RAM. The use of virtual memory instead of physical memory has a drastic negative impact on performance.

In 1998, ESRI and Data General Corporation (Peters 2003) conducted tests to determine how well Windows NT 4.0, Terminal Server Edition, would support multiple users of ArcGIS. Although their results are dated, they determined that performance scaling on multiple CPU Windows-based servers was similar to what is experienced on UNIX systems and that the ArcGIS software performed well in a Terminal Server environment, provided adequate memory is available.

It is important to note that, in 1998, they were testing the command-line portion of ArcGIS (now called Workstation Arc/Info). The newer versions of ArcGIS are COM-based and can be expected to generate more threads and require more system resources per user.

In a working environment, the U.S. Army Engineer Research and Development Center (ERDC) Environmental Laboratory (EL) commonly supports 10 concurrent users running ArcGIS on a 6-CPU (700-Mhz Pentium-III XEON processors) system with 4 GB of RAM. Each user usually is running at least one instance of ArcMap and ArcCatalog. In addition, many other applications are installed on the terminal server and are being used simultaneously.

\section{Windows and virtual memory use}

Regardless of how much memory the server has, Windows is not very efficient in the use of virtual memory. When multiple copies of applications or drivers are loaded into memory, even though large amounts of RAM may still be available, the OS makes use of lots of slow virtual memory. The result is many 
unnecessary page file read and writes which slow down the server. The ERDC EL has tested and purchased a product called TScale from KevSoft Inc. to help solve this problem. Basically, the software optimizes dynamic-linked libraries (DLL's), causing less of the application to get swapped to the page file. The result is that the server can support more users, and users recognize significant performance boosts.

The ArcGIS application, due to its architecture, is particularly susceptible to performance degradation caused by poor utilization of memory resources by the OS. Upon installing the TScale software, each instance of ArcMap and ArcCatalog recognized a savings of about $50 \mathrm{MB}$ of virtual memory.

As a district expands the use of terminal servers for GIS and other applications, it may be worthwhile to pursue the use of the TScale software, particularly when the load on terminal servers reaches a critical level.

Information about this product may be found at www.kevsoft.com.

\section{Recommendations on server selection}

Recommendations on server selection are as follows:

- Purchase as many processors as possible. The server should use XEON processors with as much processor cache memory as possible.

- The amount of system RAM is extremely important. ArcGIS requires a great deal of memory per user. As a rule of thumb, each ArcMap or ArcCatalog session will require approximately 50 to 60 MB of RAM.

- GIS applications are much more dependent on fast i/o than they are on processor speed. Purchasing the fastest RAID controllers possible is highly recommended.

\section{Configuration tips}

Configuration tips include:

- Use a RAID configuration, if possible.

- Install at lease two controllers: one to support the OS and one for applications. Even if you must use one controller, separate the OS and applications as much as possible.

- If possible, separate the applications and temporary files on separate controllers.

- Distribute hard disk access as much as possible.

- Do not install Oracle or any other services on the terminal server. Do not use the terminal server to support print serving, etc.

- All partitions must use the New Technology File System. 
- If possible, only install one network protocol on the server. This frees up system resources and reduces network traffic.

- Reserve at least $1 \mathrm{~GB}$ for user profiles.

\section{Software Licensing}

To operate in a terminal server environment, two to three types of licenses will be required (in addition to any application-specific licensing requirements):

- Microsoft Windows 2000 Server Client Access license

- Citrix MetaFrame XP connection license

And, if connecting from a non-Windows 2000 computer:

- Microsoft Windows 2000 Terminal Services Client Access license

Licenses from Microsoft and Citrix will be required for end users to connect to a Windows 2000 Terminal Server running Citrix MetaFrame. All licensing is transparent to the end user on the client device.

\section{Operating system}

Each device that initiates a Windows 2000 Terminal Services session must by licensed with the following:

- Windows 2000 Professional license or Windows 2000 Terminal Services Client Access license.

- Windows 2000 Server Client Access license or BackOffice Family Client Access license.

These licenses are required whether or not third-party software, such as Citrix Metaframe, is used.

It should be noted that users connecting to a terminal services session from a client computer running Windows 2000 do not require a Windows 2000 Terminal Services Client Access license. Users running other OS's, such as Linux, Windows 98, Windows 95, etc., will require a Terminal Services Client Access license.

Client Access licenses may be purchased in two ways: as per seat licenses, or as per server licenses. Per seat licenses are permanently assigned to specific client computers. When a user connects to a terminal services session from such a device, the server issues them a Client Access license. This license remains bound to this client and is not released when the client logs off. Per server licenses allow licensing for a set number of concurrent users and are freed up when users log off. 
More detailed information regarding Windows terminal services licensing can be found at: http://www.microsoft.com/windowsserver2003/docs/ termservlicensing.doc.

\section{Citrix Metaframe}

With the release of MetaFrame XP, Citrix changed its licensing from a server-based licensing model to a connection-based model. This means that licenses are no longer bound to specific servers as in the past. This is a benefit if the district at some point wishes to implement a terminal server farm, rather than a single terminal server.

Citrix sells three different versions of the MetaFrame software. These differences are outlined in Figure 4.

\begin{tabular}{|c|c|c|c|}
\hline & $\begin{array}{c}\text { MetaFrame } \\
\text { XPs }\end{array}$ & $\begin{array}{c}\text { MetaFrame } \\
\text { XPa }\end{array}$ & $\begin{array}{c}\text { MetaFrame } \\
\text { XPe }\end{array}$ \\
\hline \multicolumn{4}{|l|}{ Total "Net" Leverage } \\
\hline NFuse integration & - & - & - \\
\hline Support for corporate portals & - & - & - \\
\hline Application filtering and caching & - & - & - \\
\hline Web-based client installation & - & - & - \\
\hline \multicolumn{4}{|l|}{ Ultimate Flexibility } \\
\hline Seamless user experience & - & - & - \\
\hline Access to local system resources & - & - & - \\
\hline Security & - & - & - \\
\hline Universal connectivity & - & - & - \\
\hline \multicolumn{4}{|l|}{ Unparalleled Manageability \& Scale } \\
\hline Printer management & - & - & - \\
\hline Centralized license management & - & - & - \\
\hline Advanced shadowing & - & - & - \\
\hline Client management & - & - & - \\
\hline Application publishing & - & - & - \\
\hline Application-based load management & & - & - \\
\hline Application scheduling & & - & - \\
\hline Monitor system health and performance & & & - \\
\hline Perform system capacity planning & & & - \\
\hline Centrally install and uninstall applications & & & - \\
\hline Distribute service packs, updates and files & & & - \\
\hline Management access through $\mathrm{HP}$ and Tivoli & & & - \\
\hline Support for standard SNMP monitoring & & & - \\
\hline
\end{tabular}

Figure 4. Citrix Metaframe product versions (Citrix Systems, Inc. 2002)

For the typical Corps district, it is recommended that MetaFrame XPs version be purchased. MetaFrame XPa adds load management (load balancing), which would only be useful if a server farm is being implemented. Citrix Metaframe XP Starter Packs are sold with 5- and 20-user connection licenses. Additional connection licenses can be purchased in 5-, 10-, 20-, and 50-user packs.

Individual licenses are associated with current users on a system. Therefore, when a user logs out, the license is released and available to another user. Therefore, a sufficient number of MetaFrame Connection licenses must be purchased to support only the number of expected concurrent system users. 


\section{ArcGIS licensing}

Most districts have already purchased single use licenses for ESRI software products such as Arcview. These licenses are locked to individual PC's. When a district decides to pursue a terminal server implementation, it would be wise to change to a concurrent (floating) license approach. While floating licenses cost more than single use licenses, the district only has to maintain the number of licenses required to support the number of concurrent users of the software. The district can then legally install the ESRI software on as many PC's as it wants. When a user starts up an application, such as ArcGIS in ArcView mode, a request is made to a central server running the license manager software. A license is then checked out, if available, and when the user is finished with his or her session, the license is released. Since the checking out and releasing of licenses only requires a few bytes of information to go over the network, this type of licensing scheme will work regardless of whether or not the district chooses to implement a terminal server.

The only limitation of the floating licenses is that the end user system must be connected to the network in order to be able to check out a license. The district may wish to maintain a very limited number of single user licenses for systems such as laptops that will be used for travel and which may not have network access.

ESRI uses the FLEXlm software from Globetrotter Software to manage floating licenses. This software uses port number 27005 for checking out licenses. In addition, a hardware key must be installed in the parallel port of the license server.

If a decision is made to use floating licenses, it is recommended that the terminal server NOT be used as the license server. The reasons for this are discussed in the following section.

Since the terminal server and the license server should be on the same side of the firewall, there should be no need to open port 27005 .

\section{ESRI-Specific Considerations}

ESRI maintains and frequently updates guidance on the use and limitations of using their products in a terminal server environment. The ESRI document can be accessed at the following link:

http://support.esri.com/index.cfm?fa $=$ knowledgebase.whitepapers.viewPaper\&P $\underline{I D=43 \& \text { MetaID }=389}$.

The following paragraphs list some of the current limitations and considerations at the time this document was written. 


\section{Terminal server support from ESRI}

ESRI supports ArcGIS 8.x products on Windows Terminal Server at support level 3. This level of support reflects that there are some known limitations with this operating environment. The main limitations concern printing and setting the software mode through the ArcGIS Desktop Administrator. These are discussed in more detail below.

\section{ArcGIS installation on terminal servers}

As with all applications on Windows Terminal Server, new programs must be added through the Control Panel $\rightarrow$ Add/Remove Programs interface. This puts the system in "install mode."

Installing ArcGIS applications on a Windows Terminal Server is not straightforward as the installation routine makes some changes to the OS environment and then requires a reboot before proceeding with the installation. ESRI has compiled some installation tips for Windows Terminal Server (DeWeese 2002a). The following are derived from that document:

- Prior to installation:

- Only install the software through the console interface - not through a terminal session. Files are placed in temp directories specific to the session. If a reboot is required, session temp files will be deleted.

- When you set up the terminal services in application server mode, you are given a choice to set system permissions either to be compatible with Windows 2000 Users or Windows NT 4.0 Terminal Server Users. It is recommended that, unless absolutely necessary, you set permissions to be compatible with Windows NT 4.0 Terminal Server Users. If you must use Windows 2000 permissions, then you should consult the ESRI Terminal Server Installation Notes (DeWeese 2002a) for issues related to these permissions.

- It is recommended that the ESRI license manager software not reside on the terminal server. Indeed, if you are going to remap the system drive on the terminal server, it will not be possible to run the license manager software on the terminal server.

- Ensure the account you are using is part of the Administrator's group.

- Make sure you are in "install" mode. This is accomplished by installing programs through the Control Panel $\rightarrow$ Add/Remove Programs interface.

- During installation:

- During the first part of the installation, the install process may ask permission to update the OS. If you answer "yes," files will be installed and the system will have to reboot. When the reboot is complete and you log back in, you will be put back into the install 
process automatically - but the system will no longer be in "install" mode. Exit the installation and then go to the Control

Panel $\rightarrow$ Add/Remove Programs interface and reenter the installation manually.

- Post Installation:

- If Workstation ArcInfo is installed on the terminal (this will be the case only if the ArcInfo version of ArcGIS is installed on the terminal server), then changes will need to be made to the $\%$ HOMEPATH $\%$ environment variable. Workstation ArcInfo does not like spaces in directory names and the default for Windows 2000 is set to the path Document and Settings $\backslash \%$ USERNAME\%. You can fix this by modifying the user's home directory using the Terminal Server Profile tab under the Computer Management tool. The name should be changed to the DOS-naming convention using a " $\sim$ character.

- By default, Citrix will share a server session connection when two or more seamless applications are launched. This causes the environment setting to be different for subsequent "seamlessly launched" applications, including changing the TMP and TEMP directories to C: IWINNT $\sim$ Temp. This causes abnormal behavior with applications such as ArcGIS, including the inability to write to the TMP and TEMP directories. To avoid this potential problem, Citrix session sharing should be disabled when running ArcGIS. The fix is to add a "SeamlessFlags" DWORD with a value of "l" to the following registry key:

- Registry Key: HKeyLocalMachine SYSTEl CurrentControlSetl Control $\backslash$ Citrix $\backslash W$ fshell $\backslash$ TWI

- New DWORD value: SeamlessFlags

- Value: 1

\section{Specific ArcGIS limitations}

Early releases of terminal server technology possessed a number of limitations, many of which were specific to individual applications, such as ESRI's GIS applications. With each new release of the technology more and more of these limitations have been overcome. Today, there are really only a few specific limitations that must be addressed in serving the ArcGIS applications through a terminal server.

\section{Software mode setting}

Versions of ArcGIS prior to 8.3. For versions of ArcGIS prior to 8.3, there was no way to set a software mode (ArcView, ArcEditor, or ArcInfo) for individual user sessions. Since at the ArcGIS 8.x level ArcInfo, ArcEditor, and ArcView all share the same architecture, it was impossible for different terminal server clients to run different modes of the software. When the Desktop 
Administrator application is used to set the mode of software - this setting affects ALL users on the system. According to ESRI this is a hard limitation, with no workaround; however, ArcView 3.x and ArcView8.x can both be running at the same time since they do not share the same architecture.

Since this setting affects all users on the terminal server, access to the Desktop Administrator should be restricted to the Administrator user.

Since this is such a hard limitation, it may be that power users at the district office may wish to use the ArcInfo mode of the software on their local PC's, as they currently do, and that the ArcView version of the software can be served on the terminal server to users at the district.

ArcGIS version 8.3. In version 8.3, ESRI implemented a new ESRI_SOFTWARE_CLASS environment variable that can be used to specify a level of the product to be used for individual user sessions. This variable can be set in the user's profile or can be set using a batch file. The setting of this variable overrides the mode set in the Desktop Administrator application and allows each user to utilize the appropriate level of the software for their needs. There are, however, several small limitations.

- Due to the way ArcToolBox DLL's are registered during the installation process, the Desktop Administrator should be configured to the highest level of license the organization possesses. If the Desktop Administrator application is used to set the mode of software operation all users are still affected, unless the ESRI_SOFTWARE_CLASS is used to override this setting.

- The ArcMap title bar usually displays the mode in which the software is running (i.e., "ArcMap - ArcInfo" or "ArcMap - ArcView"). When the ESRI_SOFTWARE_CLASS environment variable is used to set the mode the user is running, the title bar does not reflect this setting. For example, even though a user may be operating at the ArcView level of license, the title bar will still display the highest level of product installed on the server. This may be confusing to the user, and there is no workaround for this problem.

\section{Printing}

Printing represents probably the most significant challenge to the successful implementation of a terminal server for geospatial applications. Users at the remote offices need to be able to locally print large plot files. These plots often contain a great deal of raster imagery. It is not unusual for GIS plot files to reach 60 or even $100 \mathrm{MB}$ in size. Obviously, if the plot files must traverse the network from the terminal server to the client's local printer, this can cause serious problems with network bandwidth.

One potential solution is to use RIP software such as Image Printer from Handmade Software or ESRI's ArcPress to translate the plot files from Encapsulated Postscript (which are very, very large) into native printer language, 
such as RTL used by Hewlett-Packard large format plotters. Users would plot their large map documents to files on the terminal server. Then RIP software would do the translation to RTL and send the much smaller file over the network to the plotter. Since RTL is the native language of the plotter, no translation would need to be done on the plotter and the file would begin to print immediately.

Echevarria (2002) specifically addresses the issue of plotting in a terminal services environment.

\section{Digitizing}

ArcGIS uses Wintab drivers to support digitizing in the ArcMap desktop application. These drivers do not work in a Windows Terminal Server environment at this time. ESRI is working with the Wintab driver providers to determine a solution. This affects users who wish to digitize using a digitizer table. On-screen (or "heads-up") digitizing using a mouse on the screen does not require Wintab drivers and works in a Windows Terminal Server environment.

The ArcInfo workstation software does not use the Wintab drivers, but does not work in a Windows Terminal Server environment.

In summary, at this point, users who wish to digitize using a digitizing tablet need to have the ArcGIS software loaded on their local PC.

\section{Security}

Currently, field offices in most Corps districts are located on the same side of the firewall as the resources in the main district office. Therefore, the setup and configuration of the terminal server should be just like any other server within the district, and no special provisions need to be made for a firewall.

Should the firewall situation be different, Citrix provides many options for securing a Citrix MetaFrame XP server. The following is a basic list of the options.

\section{Firewall considerations}

The default port on MetaFrame servers for independent computing architecture (ICA) sessions is 1494. This port must be open on firewalls for inbound communication if ICA clients are outside the firewall. The port used on the client for the ICA session is configured dynamically when the session is established.

The Network Protocol setting specified for server location in the ICA client affects the following deployment issues related to ICA browsing:

- The communications protocol the client uses to locate servers. 
- The Citrix component the client communicates with.

- The port the client communicates with.

- The default locations the client contacts.

Citrix recommends that ICA clients use TCP/IP+HTTP for ICA browsing. Among other advantages, this protocol does not use user datagram protocol or broadcasts to locate terminal servers. To use the TCP/IP+HTTP protocol with clients outside a firewall, configure the firewall to pass inbound HTTP packets on port 80, the default port for the Citrix XML service on MetaFrame XP servers. This port is usually open on firewalls for inbound HTTP packets to Web servers.

In ICA sessions, ICA clients communicate with port 1494 on MetaFrame servers. If the clients are outside the firewall, this port must be open for inbound communication to MetaFrame servers (Figure 5).

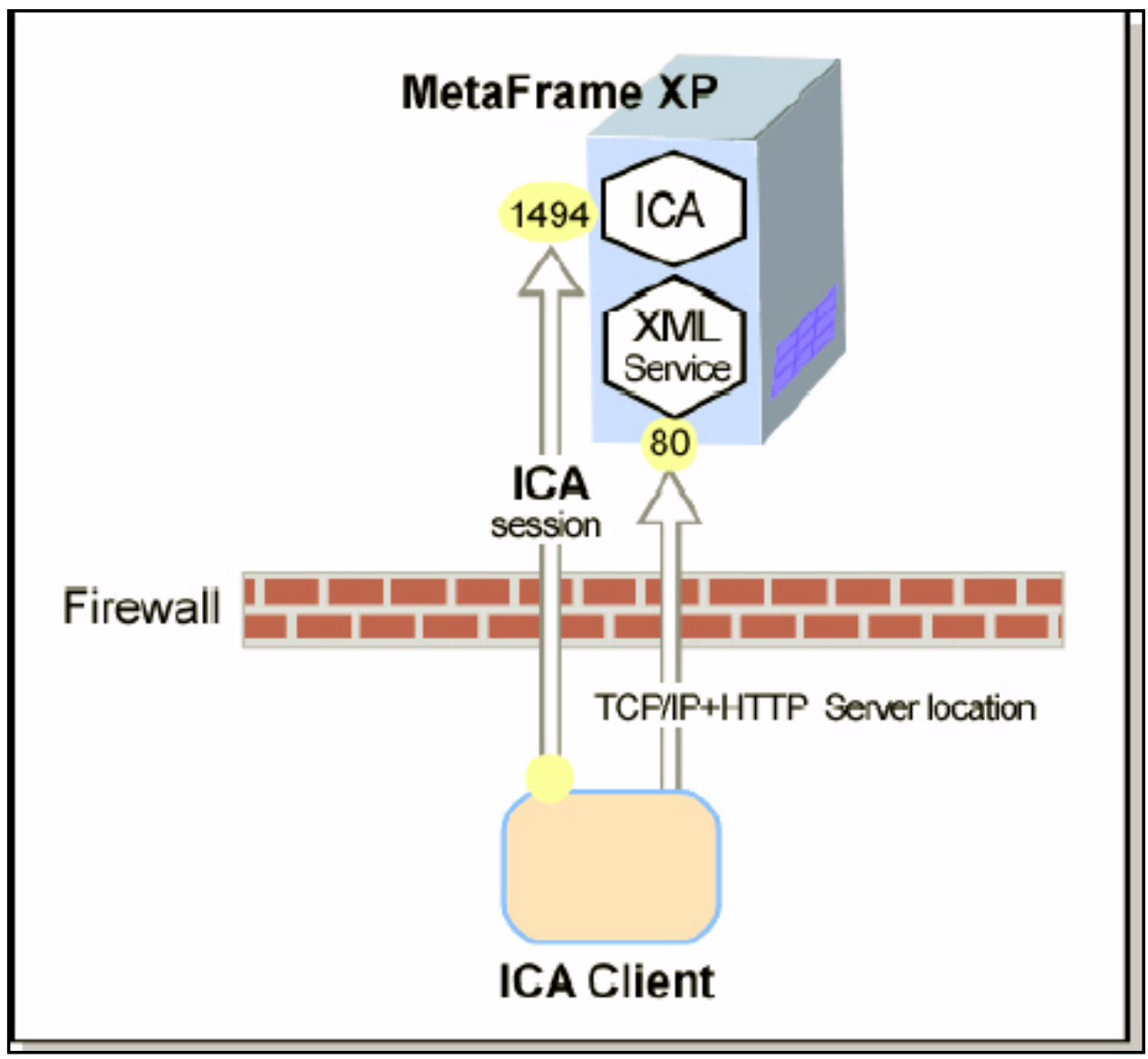

Figure 5. Basic client/server communication (Citrix Systems, Inc. 2002)

The process of running a session is outlined below:

- The client sends a request to the Citrix XML service on port 80 on a specified server using HTTP.

- The XML service sends the address of a server that has the requested application. 
- The ICA client establishes an ICA session with the MetaFrame XP server specified by the XML service. ICA packets travel from the client to port 1494 on the server. ICA packets travel from the server to a dynamically assigned port number on the client.

Figure 6 illustrates the process if a demilitarized zone is set up.

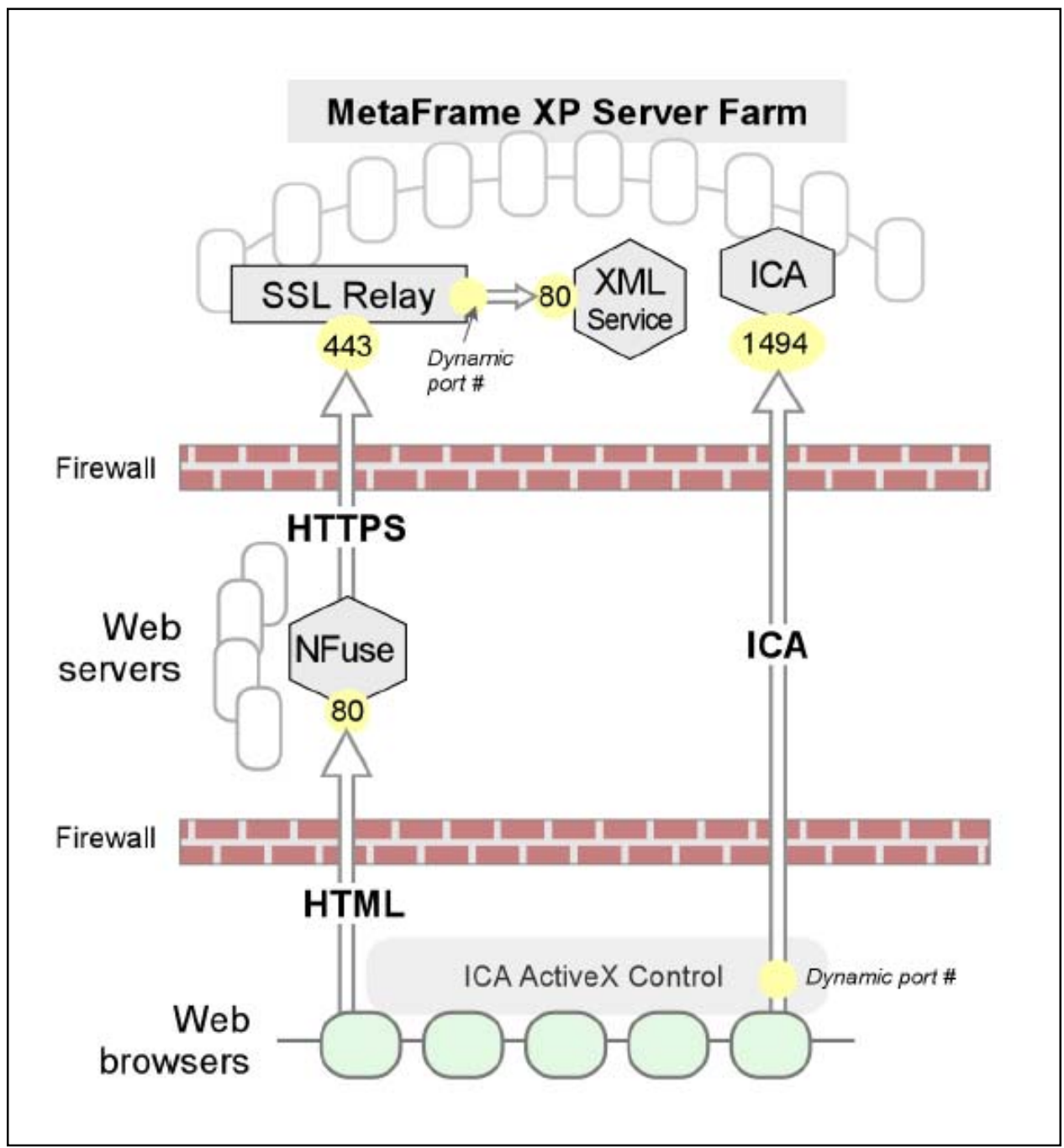

Figure 6. Communication with NFuse-enabled Web server (Citrix Systems, Inc. 2002)

In a network configuration with Web servers in a demilitarized zone between firewalls, users' Web browsers send application requests to NFuse-enabled Web servers. Web servers send secure (HTTPS) requests to the Secure Sockets Layer (SSL) relay and XML service in the server farm.

ICA clients establish ICA sessions with MetaFrame XP servers on port 1494. The port used on the clients is configured dynamically.

If SSL is used, the process is as shown in Figure 7. 


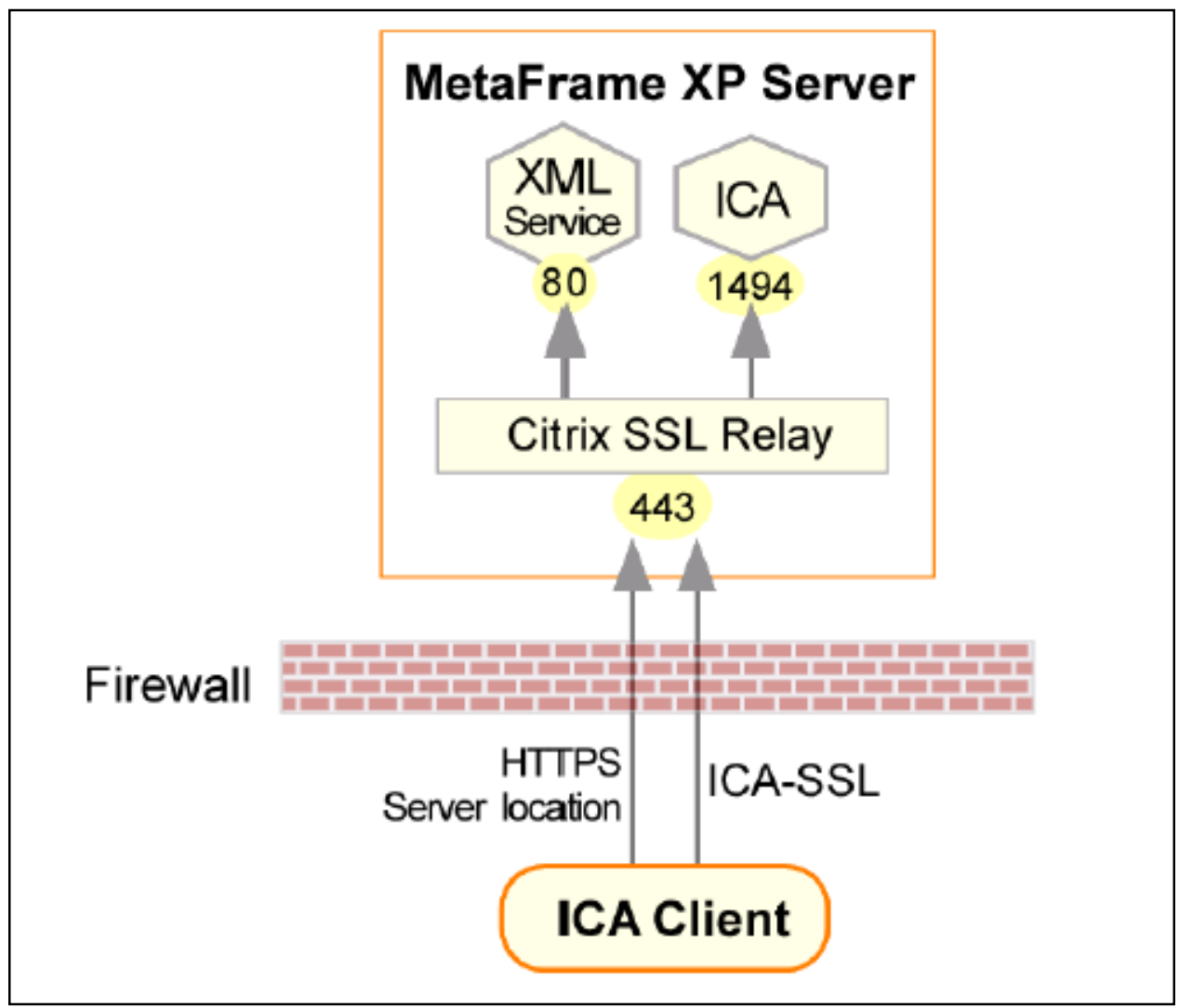

Figure 7. Client to server communication with SSL (Citrix Systems, Inc. 2002)

For SSL communication, port 443 is open for inbound communication to the Citrix SSL relay. The client communicates with the SSL relay for server location and ICA session communication.

\section{FIPS-140 requirements}

When deploying Citrix MetaFrame XP in an environment where FIPS-140 requirements need to be met, Feature Release 2 of the software needs to be loaded. This adds support for transport layer security (TLS), the latest standardized version of SSL. With Feature Release 2, Citrix supports cryptographic modules that are FIPS-140 validated. With this release Smart Cards, IPSec, and Government Cyphersuite are supported as well.

Citrix Systems, Inc. (2002) should be consulted for more information regarding FIPS-140 level security considerations. 


\section{References}

Citrix Systems, Inc. (2002). "Citrix MetaFrame XP security standards and deployment scenarios," Citrix Systems, Ft. Lauderdale, FL, http://www. citrix.com/products/library/MetaFrame_XP_FR2_Security_Standards.pdf.

Deweese, J. (1998). “Windows Terminal Server scaling tests," ESRI Technology Portal, http://eslims.esri.com/miscfiles/DG_Test_Report.PDF (7 July 1998).

. (2002a). "Windows 2000 server terminal services/Citrix installation tips," ESRI Technology Portal, http://eslims.esri.com/miscfiles/ W2K_WTS_Installation_Tips.pdf(9 Sep 2002). . (2002b). "WTS/Citrix support," ESRI Technology Portal, http://eslims.esri.com/wtssupport.htm (9 Sep 2002).

Echevarria, R. (2002). "Citrix MetaFrame printing considerations for ArcGIS," ESRI Systems Integration Technical Paper, Environmental Systems Research Institute, Redlands, CA, http://eslims.esri.com/miscfiles/Citrix_Printing.pdf.

Harwood, T. (2002). Inside Citrix ${ }^{\circledR}$ MetaFrame XPTM: A system administrator's guide to Citrix MetaFrame $\mathrm{XP} / 1.8^{\mathrm{TM}}$ and Windows ${ }^{\circledR}$ terminal services, Addison-Wesley, Boston, MA.

Levinsohn, A.G. (2000). "Stirring the spatial soup," Geoplace.com, http://www.geoplace.com/ge/2000/0400/0400dt.asp

Mathewson, M. (1999). "What protocol is right for you?" ThinPlanet.com, http://www.thinplanet.com/opinion/protocols.asp\#SeamlessWindows (April 1999).

Microsoft. (2000). "Microsoft Windows 2000 terminal services, licensing technology white paper," Microsoft Corporation, Redlands, WA, http://www.microsoft.com/WINDOWS2000/techinfo/howitworks/terminal/tsli censing.asp

Nieh, J., Yang, S.J., and Novik, N. (2000). "A comparison of thin-client computing architectures," Technical Report CUCS-022-00, Columbia University, Network Computing Laboratory, http://www.ncl.cs.columbia. edu/publications/cucs-022-00.pdf 
Peters (2003). "System design strategies," ESRI White Paper, Environmental Systems Research Institute, Redlands, CA, http://www.esri.com/library/ whitepapers/pdfs/sysdesig.pdf. 


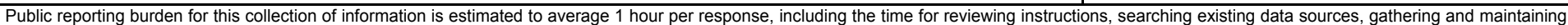

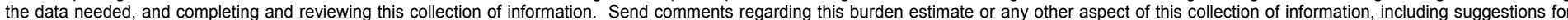

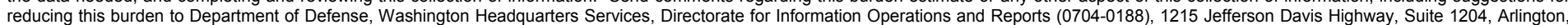

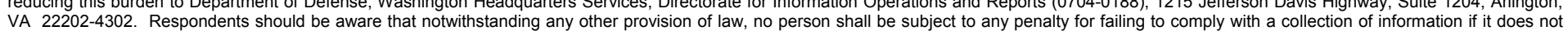
VA 22202-4302. Respondents should be aware that notwithstanding any other provision of law, no person shall be $s$.
display a currently valid OMB control number. PLEASE DO NOT RETURN YOUR FORM TO THE ABOVE ADDRESS.

\begin{tabular}{l|c}
$\begin{array}{l}\text { 1. REPORT DATE (DD-MM-YYYY) } \\
\text { August } 2003\end{array}$ & $\begin{array}{c}\text { 2. REPORT TYPE } \\
\text { Final report }\end{array}$ \\
\hline
\end{tabular}

\section{TITLE AND SUBTITLE}

Using Terminal Services to Serve Geospatial Software and Data Resources to Corps

Project Offices

3. DATES COVERED (From - To)

5a. CONTRACT NUMBER

5b. GRANT NUMBER

5c. PROGRAM ELEMENT NUMBER

6. AUTHOR(S)

Mark R. Graves

7. PERFORMING ORGANIZATION NAME(S) AND ADDRESS(ES)

U.S. Army Engineer Research and Development Center

Environmental Laboratory

3909 Halls Ferry Road

Vicksburg, MS 39180-6199

9. SPONSORING / MONITORING AGENCY NAME(S) AND ADDRESS(ES)

U.S. Army Corps of Engineers

Washington, DC 20314-1000 5d. PROJECT NUMBER

5e. TASK NUMBER

5f. WORK UNIT NUMBER

8. PERFORMING ORGANIZATION REPORT NUMBER

ERDC/EL TR-03-13

10. SPONSOR/MONITOR'S ACRONYM(S)

11. SPONSOR/MONITOR'S REPORT NUMBER(S)

\section{DISTRIBUTION / AVAILABILITY STATEMENT}

Approved for public release; distribution is unlimited.

\section{SUPPLEMENTARY NOTES}

\section{ABSTRACT}

Advances in geographic information systems (GIS) are causing the technology to no longer be considered a separate entity, but rather an integral component of the overall information technology infrastructure. Most GIS platforms are moving from simple file-based data structures to complex spatial geodatabases built within large-scale database platforms such as Oracle. The move toward centralized databases has many ramifications for how geospatial tools and data are distributed throughout a complex organization such as the Corps of Engineers. Project offices, in particular, represent a special challenge due to the limitations of their network connections to the district offices. Windows Terminal Server technology represents one solution for serving these resources to project and field offices. This document addresses some of the technical issues related to the use of this technology and some of the advantages and limitations of such an approach.

\section{SUBJECT TERMS}

Citrix Metaframe

Geographic information systems (GIS)

16. SECURITY CLASSIFICATION OF:

\begin{tabular}{|l|l|}
\hline a. REPORT & b. ABSTRACT \\
UNCLASSIFIED & UNCLASSIFIED \\
\hline
\end{tabular}

Geospatial

Network

Systems administration

\begin{tabular}{|l|c|c|}
\cline { 1 - 1 } \begin{tabular}{l|l} 
c. THIS PAGE \\
UNCLASSIFIED
\end{tabular} & $\begin{array}{l}\text { 17. LIMITATION } \\
\text { OF ABSTRACT }\end{array}$ & $\begin{array}{c}\text { 18. NUMBER } \\
\text { OF PAGES }\end{array}$ \\
\cline { 1 - 1 } & & 27 \\
\hline
\end{tabular}

Windows Terminal Server

19a. NAME OF RESPONSIBLE PERSON

19b. TELEPHONE NUMBER (include area code) 